Check for updates

Cite this: RSC Adv., 2017, 7, 49455

\title{
Microfluidic fabrication of hollow protein microcapsules for rate-controlled release $\uparrow$
}

\begin{abstract}
Yiming Feng (D) and Youngsoo Lee*
Droplet-based microfluidics is an emerging technique that is capable of producing sophisticated supramolecular microcapsules in one step. However, food materials, due to their physical and chemical complexity, have limited success with microfluidic processes. The objectives of this work were to produce food-grade protein microcapsules in a microfluidic system and to control their structural properties by adjusting the formulation and flow rates. In this study, a T-junction microfluidic chip was used to create an interface for zein to self-assemble, and therefore form microcapsules with tunable particle sizes, pore distributions, and permeabilities. Our SEM and CLSM results show that the particle size and number of pores increased with the flow rate of the dispersing phase, while the pore size decreased with the flow rate of the dispersing phase. In order to quantify the release profiles, the release half-life $\left(t_{50}\right)$ was used as an indicator for the particle permeability. A wide range of $t_{50}$, from 3 to 62 minutes, was achieved by changing the zein concentration and flow rate of the dispersing phase. Using Rhodamine $B$ as an encapsulant, the release rate was positively correlated with the zein concentration and flow rate of the dispersing phase. Finally, response surface analysis of the formulation and flow rate was applied to aid the design of a carrier with a desirable release rate.
\end{abstract}

Received 4th August 2017

Accepted 16th October 2017

rsc.li/rsc-advances
Droplet-based microfluidic platforms are an emerging technique that have become a topic of interest for analytical (e.g. cell analysis, DNA assays and high-throughput screening) ${ }^{7}$ and synthetic (e.g. encapsulation, polymerization and anti-solvent nanoparticle fabrication $)^{\mathbf{8 - 1 0}}$ applications. For encapsulation applications, microfluidic platforms provide precise particle size control over a wide range, which is vital for ensuring product homogeneity. ${ }^{8}$ Microfluidic platforms have been reported for single-step processes for fabricating many sophisticated supramolecular microcapsules, for example, nonspherical particles, ${ }^{11}$ porous microcapsules, ${ }^{12}$ alginate microspheres, ${ }^{13}$ dendritic microcapsules, ${ }^{14}$ cross-linked protein capsules ${ }^{15}$ and biological microgels. ${ }^{16}$ Microfluidic chips have also been deployed to trigger precisely controlled interfacial reactions for fabricating complex and multiple emulsions. ${ }^{17,18}$ However, most of these approaches require crosslinking agents, UV treatment, and polymer precursors, which may be toxic and potentially cytotoxic. These limitations are especially unfavorable for encapsulating live cells, as well as sensitive pharmaceuticals and nutraceuticals. ${ }^{8}$ Furthermore, there have been very few successes in using biological materials such as proteins in microfluidic processes as they could be prone to clogging the microfluidic channels.

Zein is a storage protein from corn that is insoluble in water but capable of self-assembling to form many types of microstructure such as films, ${ }^{19}$ nanoparticles ${ }^{20,21}$ or Pickering emulsions. ${ }^{22}$ Its distinct amino acid composition and rigidity in the solid state make it a potential food-grade wall material for
Department of Food Science and Human Nutrition, University of Illinois at Urbana-Champaign, 1304 W Pennsylvania Ave., Urbana, Illinois, 61801, USA. E-mail: leeys@illinois.edu

$\dagger$ Electronic supplementary information (ESI) available. See DOI: 10.1039/c7ra08645h 
fabricating diffusion-driven controlled-release systems. To date, self-assembly of zein could only be achieved in an uncontrolled or limited control manner (e.g., evaporation induced selfassembly $^{23}$ or anti-solvent self-assembly ${ }^{24}$ ). However, recent advances have made it possible to direct particle assembly using microfluidic platforms. ${ }^{25}$ Many studies have explored the application of zein as a delivery carrier, although several limitations have been reported as well. ${ }^{26-28}$ One inherent limitation is that current zein-based delivery systems, in which encapsulation is achieved during a co-precipitation of zein and the encapsulant in water, are only able to carry hydrophobic compounds. Therefore, the encapsulant has to be insoluble in water but soluble in $60-90 \%$ ethanol. The second limitation is associated with particle size control in that only submicron $(100-300 \mathrm{~nm})$ zein nanoparticles are able to be generated. ${ }^{24}$ Small particles generally release encapsulated compounds rapidly due to their high surface-to-volume ratios and instant particle disintegration in the gastro-intestinal (GI) tract, thus they are not feasible for sustained release. ${ }^{29}$ In this study we report a facile method to fabricate hollow microcapsules with tunable release rates using a food-grade protein, zein. To the author's knowledge, this is the first approach creating an interface to direct zein self-assembly and form a rigid shell. A water-soluble dye, Rhodamine B, was used as a tracer to indicate the permeability of the microcapsule and analyze the release rate.

\section{Experimental}

\section{Materials}

Zein (Z3625, Sigma-Aldrich, St. Louis, MO) dissolved in 70\% (v/ v) ethanol (200 Proof, Decon Laboratories, King of Prussia, PA) was used as the dispersing phase. Tributyrin (W222305, SigmaAldrich, St. Louis, MO) containing $2 \%(\mathrm{w} / \mathrm{v})$ soy lecithin (MP Biomedicals Inc, Solon, $\mathrm{OH}$ ) was used as the continuous phase. Rhodamine B (R6626, Sigma-Aldrich, St. Louis, MO) was added into the dispersing phase at $2 \mathrm{mg} \mathrm{ml}^{-1}$ to assess the rate of release. Hexane (Macron Fine Chemicals, Center Valley, PA) was used to wash the microcapsules. No. 42 (pore size $2.5 \mu \mathrm{m}$ ) Whatman filter paper (Whatman Inc., Florham Park, NJ) was used to collect the final products.

\section{Microfluidic fabrication}

The dispersing phase was prepared by dissolving various amounts $(2 \%, 4 \%$ and $6 \% \mathrm{w} / \mathrm{v})$ of zein into an ethanol-water binary system containing $70 \%(\mathrm{v} / \mathrm{v})$ ethanol. The continuous phase was prepared by mixing $2 \%(\mathrm{w} / \mathrm{v})$ of soy lecithin with tributyrin by stirring at $250 \mathrm{rpm}$ overnight, followed by centrifugation at $2000 \mathrm{~g}$ for $10 \mathrm{~min}$ to remove impurities. Hollow zein microcapsules were then produced in a $100 \mu \mathrm{m}$ T-junction microfluidic chip (Dolomite, UK) through internal phase separation and the mechanism is shown in Fig. 1. The samples prepared in this study are listed in Table 1 . Three flow rate combinations were chosen for this study based on our preliminary study. Flow rates were controlled using a Harvard Model 11 Elite syringe pump (Harvard Apparatus Inc., Holliston, MA).
An amount of $2 \mathrm{mg} \mathrm{ml}^{-1}$ of Rhodamine B (Sigma-Aldrich, St. Louis, MO) was added into the dispersing phase as a release model indicator. Once collected, the capsules were immediately washed with hexane three times on a filter paper under vacuum. The final microcapsules were obtained by transferring the filtered samples into a convective oven at $30{ }^{\circ} \mathrm{C}$ for 6 hours to remove the excess water.

\section{Characterization of microcapsules}

Scanning electron microscopy (SEM). The surface morphology of the final products was imaged using a Quanta 450 FEG environmental scanning electron microscope (ESEM, FEI Company, Hillsboro, OR) located at the Beckman Institute. The blocks were mounted on SEM stubs and coated with $60 \mathrm{~nm}$ of gold-palladium using a Desk-II turbo sputter coater (Denton Vacuum, Moorestown, NJ). Flash-dry colloidal silver paint (cat. no. 04998-AB; SPI Supplies, West Chester PA) was applied to the blocks to ensure proper grounding. The embedded particles were then imaged using a Quanta 450 FEG environmental scanning electron microscope (ESEM; FEI Company, Hillsboro, OR). Image analysis was then performed using Image-Pro Plus 7.0 software (Media Cybernetics, MD, USA) to calculate the particle size of each sample.

Confocal laser scanning microscopy (CLSM). CLSM was used to visualize the internal structure of the microcapsules. Freshly collected microcapsule suspensions $(1 \mathrm{ml})$ were stained with 40 $\mu \mathrm{l}$ of Nile blue $(0.1 \%)$ for $2 \mathrm{~h}$. A Zeiss LSM 700 confocal microscope (Zeiss, Germany) was operated using an excitation wavelength of $488 \mathrm{~nm}$ for the Nile blue dye to visualize the emulsions. Images were taken at a magnification of $20 \times$ and further processed using the instrumental software.

X-ray diffraction (XRD). XRD patterns were assessed using a diffractometer (D5000, Siemens) with $\mathrm{Cu}-\mathrm{K} \alpha$ radiation $(\lambda=$ $1.5406 \AA$ ) and a $0.2^{\circ}$ step scan from 5 to $35^{\circ}$ in $2 \theta$ angles. The scanning rate was set at $1^{\circ} \min ^{-1}$. The $d$-spacing for a given scattering angle, $2 \theta$, was calculated using Bragg's law (1).

$$
d=\frac{\lambda}{2 \sin \theta}
$$

where $\lambda$ is the wavelength of the $\mathrm{Cu}-\mathrm{K} \alpha$ radiation.

Circular dichroism spectroscopy (CD). The CD spectra were recorded with a JASCO J-715 spectropolarimeter (Jasco Inc., Easton, MD). Samples were placed in a quartz cell with a $1 \mathrm{~cm}$ path length. The measurements were conducted over the range of 200-250 nm at $25{ }^{\circ} \mathrm{C}$, with a scanning speed of $50 \mathrm{~nm} \mathrm{~min}{ }^{-1}$, and a resolution of $1 \mathrm{~nm}$. The $\alpha$-helix, $\beta$-sheet, and random coil content was calculated using the BeStSel software, ${ }^{30}$ which can be accessed online at http://bestsel.elte.hu. The Savitsky-Golay smoothing algorithm was used, with a polynomial order of 3 and a smoothing window of 20 points, according to the literature. ${ }^{31}$

\section{Release profiles}

Release curves. To study the release profile, approximately $5 \mathrm{mg}$ of microcapsules was dispersed in $50 \mathrm{ml}$ of deionized (DI) water with constant stirring at $250 \mathrm{rpm}$ to release the water- 


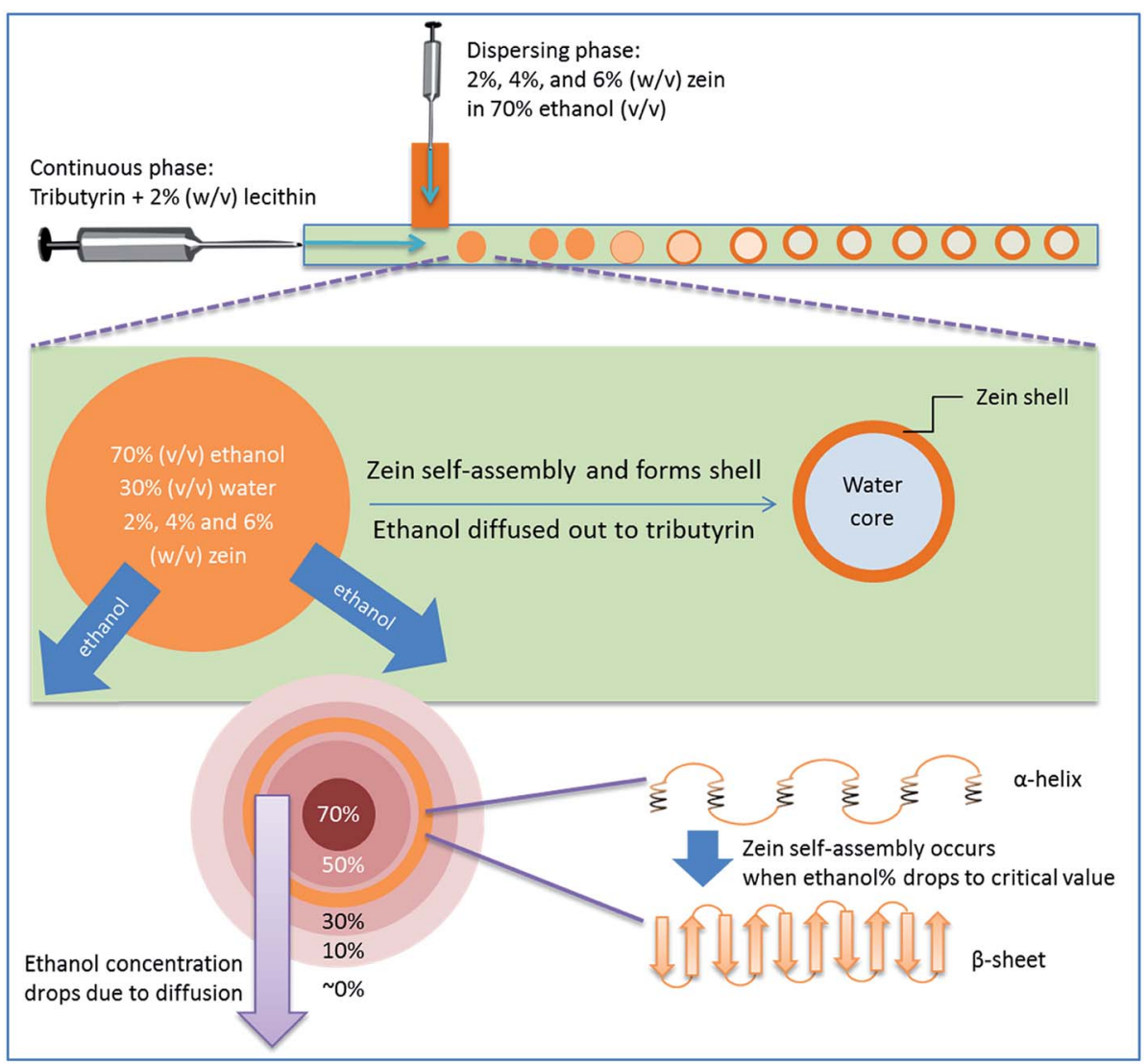

Fig. 1 A schematic diagram illustrating the mechanism of the formation of the hollow zein microcapsule in a microfluidic chip. An oil-water interface is created to initiate zein self-assembly followed by internal phase separation.

soluble dye, Rhodamine B. In total, twelve measurements were taken at 1, 3, 5, 10, 20, 30, 45, 60, 120, 180, 360, and $1440 \mathrm{~min}$, respectively, to evaluate the rate of release by measuring the dye concentration in water. For each measurement, $0.5 \mathrm{ml}$ of the dispersion was removed using a $3 \mathrm{ml}$ disposable syringe (Exelint International Co., Los Angeles, CA), and the aliquot was then filtered using a $4 \mathrm{~mm}$ syringe filter with pore size $0.45 \mu \mathrm{m}$ (Restek Corp., Bellefonte, PA), to avoid potential contamination by the microcapsules. An equivalent volume of DI water was supplemented ${ }^{32}$ upon the removal of the sample. The fluorescence intensity of the aliquot was measured using a SpectraMax M2e microplate reader (Molecular Devices, Sunnyvale, CA) in triplicate. The excitation wavelength and emission wavelength were set at $420 \mathrm{~nm}$ and $565 \mathrm{~nm}$, respectively. ${ }^{33}$ The fraction of cumulative release versus time was normalized and plotted with the assumption that complete release is achieved at 24 hours.

Release curve fitting and release half-lives $\left(t_{50}\right)$. To analyze the release kinetics, the Korsmeyer-Peppas model was used to fit the release curve $(0 \leq y \leq 0.6)^{34}$ using Matlab software R2014a (The Mathworks Inc., Natick, MA), and the fitting equation is shown below as eqn (2).

$$
\frac{M_{t}}{M_{\infty}}=k t^{n}
$$

Table 1 Summary of the sample codes and formulations

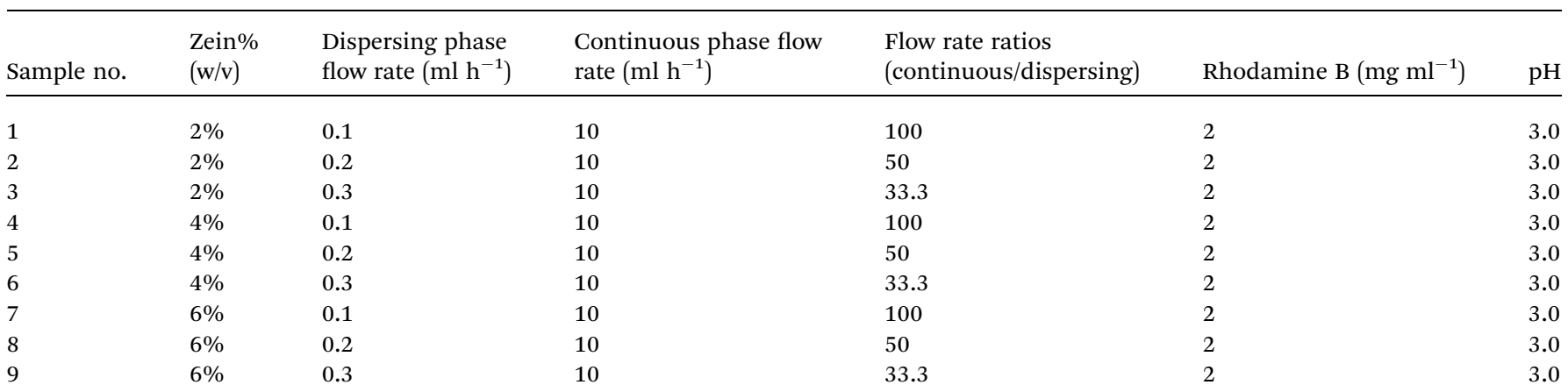




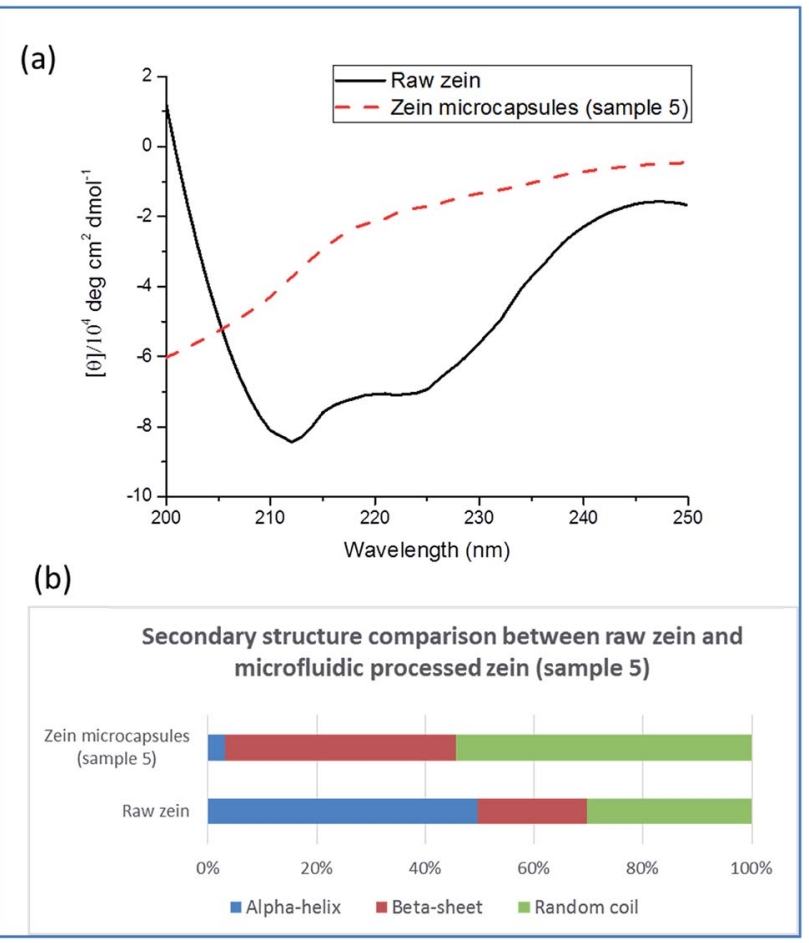

Fig. 2 (a) CD spectra of raw zein and zein microcapsules. (b) The secondary protein structure of raw zein and zein microcapsules.

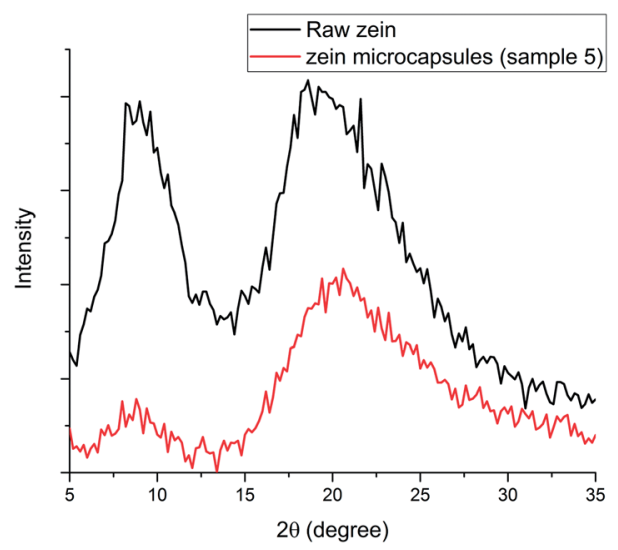

Fig. 3 XRD patterns of raw zein and zein microcapsules.

where $M_{\mathrm{t}} / M_{\infty}$ is the fractional release of Rhodamine $\mathrm{B}, t$ is the release time, $k$ is a kinetic constant characteristic, and $n$ is an exponent which characterizes the mechanism of release of the tracer. The release half-lives $\left(t_{50}\right)$ were then derived from the fitting equation. The variation of the release half-lives with the processing conditions was subjected to statistical analysis using a response surface methodology by Matlab.

\section{Results and discussion}

\section{Mechanisms of microcapsule formation}

Fig. 1 is a schematic diagram displaying the formation of hollow zein microcapsules in a T-junction microfluidic chip. A 70\% (v/ v) ethanol solution containing zein and Rhodamine B was introduced to the microfluidic channel as the dispersing phase, and the continuous phase was composed of tributyrin containing $2 \%(\mathrm{w} / \mathrm{v})$ soy lecithin as an emulsifier. Upon mixing at the joint, a jetting regime was observed where the dispersing phase maintained parallel flow before breaking up into droplets within the flow rates tested in this study. The formation of different regimes is determined by two competing forces: the viscous dragging force and surface tension. ${ }^{35,36}$ Herein, we used the jetting regime to generate particles considering its high yield and decreased risk of clogging at the expense of losing a certain degree of monodispersity in the particle size compared to that of the dripping regime.

Ethanol in the dispersing phase is miscible with tributyrin in the continuous phase, while water is not. In this study, the flow rate of the continuous phase was much higher (more than 30 times higher) than the flow rate of the dispersing phase, such that the ethanol in the dispersing phase would constantly diffuse into the continuous phase due to the concentration gradient. Once the dispersing droplets dispatch from the parallel flow, a greater interface area is created which further accelerates ethanol migration. At the same time, zein selfassembly is triggered by the continuous decrease in ethanol concentration inside the droplet. The formation of walls starts at the droplet interface, where the ethanol concentration is lower than that in the center of the droplet because of the ethanol flux. Eventually, the liquid remaining in the droplet will be mostly water, and zein becomes solid as the wall material. The selfassembly of zein is a complex process, which involves a transformation from $\alpha$-helices to $\beta$-sheets, followed by side-by-side packing of the $\beta$-sheets to form a rigid structure. ${ }^{23}$ Typically, this process is induced by evaporation of ethanol to produce a film structure. In this work, an oil-water interface was created to direct zein self-assembly in droplet microfluidic chips. The self-assembly in the internal phase separation process was verified by $\mathrm{CD}$ spectroscopy and XRD analysis. The CD spectra presented in Fig. 2 compare the secondary structures of raw zein and microfluidic-treated zein, using sample 5 as an example. Diminishment of the $\alpha$-helix structure and formation of the $\beta$ sheet structure were observed, which indicates that selfassembly of zein could occur in a short time, on the scale of a few seconds. Secondary structure is an important property for the microcapsules, and it might also affect the release profiles, though no studies have been reported yet. In terms of the secondary structure formation of zein, it is highly related to the residence time of zein self-assembly. Typically the time needed to make a difference for zein is in the scale of hours. ${ }^{23}$ However, the residence time of microfluidic processes is short, usually in the scale of seconds. So similar secondary structures between the particles were expected in this study. In order to verify this, three samples with different zein concentrations and dispersing phase flow rates were selected to analyse their CD spectra. As expected, the differences between their secondary structures were minor. More details can be found in the ESI. $\dagger$

The XRD patterns shown in Fig. 3 also compared the structures of the raw zein and zein microcapsules (sample 5). For both samples, there are two distinctive peaks exhibited at the 


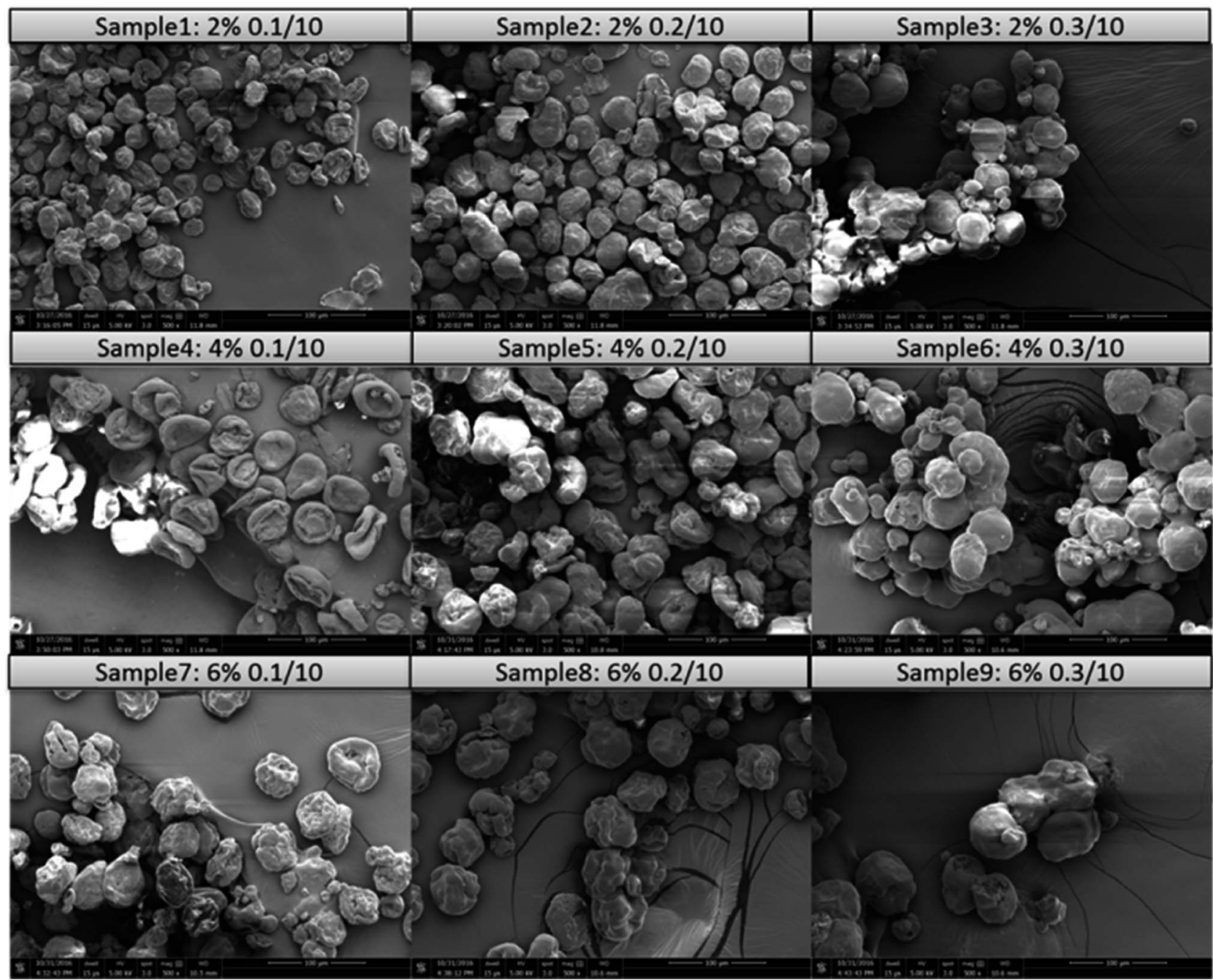

Fig. 4 The morphologies of hollow zein microcapsules produced at different concentrations of zein and flow rate combinations. Sample code: zein\% dispersing phase flow rate $\left(\mathrm{ml} \mathrm{h}^{-1}\right)$ /continuous phase flow rate $\left(\mathrm{ml} \mathrm{h}^{-1}\right)$. The scale bar for each image is $100 \mu \mathrm{m}$.

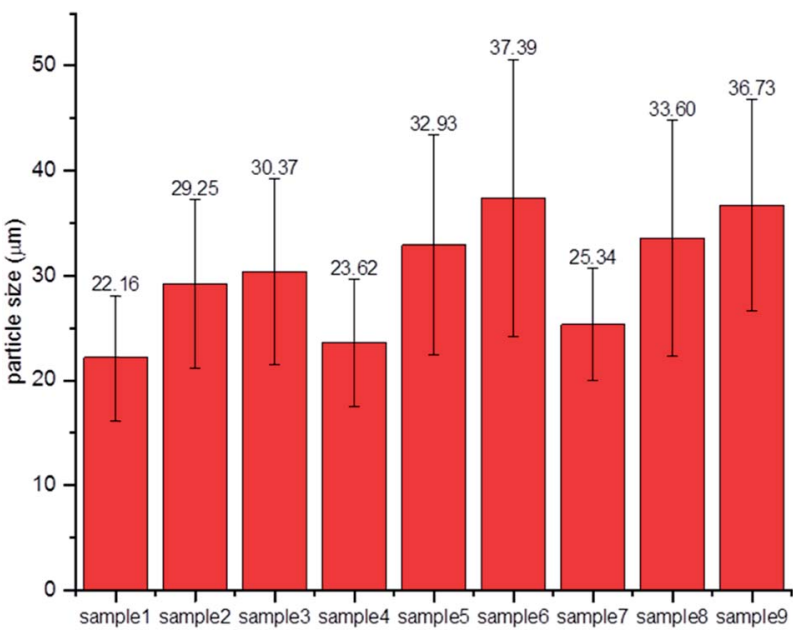

Fig. 5 Mean particle size of microcapsules using image analysis on SEM images. same $2 \theta$ angles, which are close to $9^{\circ}$ and $21^{\circ}$. Based on the $d$ spacing eqn (1), the characteristic $d$-spacing values could be calculated as $9.8 \AA$ and $4.2 \AA$, respectively. According to the literature, these two characteristic $d$-spacing values have been clearly interpreted. The smaller $d$-spacing $(4.2 \AA)$ is related to the backbone distance within the $\alpha$-helix, whereas the longer $d$ spacing $(9.8 \AA)$ comes from the lateral $\alpha$-helix packing. ${ }^{37}$ The ratio between these two peaks is an indication of the degree of $\alpha$-helix packing. ${ }^{37,38}$ Physical and chemical changes, such as thermal treatment and anti-solvent processes like that in this study, could induce secondary structural transformation. One notable and common transition is the helix-to-coil transition, ${ }^{39}$ which we also found in this study. Since the unwinding of $\alpha$ helices is a necessary step in helix-to-coil transition, the peak ratio evolution before and after microfluidic treatment is therefore an important sign of helix-to-coil transition and secondary structural transformation. In this study, it was found that the lateral $\alpha$-helix packing structure decreased in the zein microcapsules, indicating the occurrence of structural transformation (Fig. 3). 


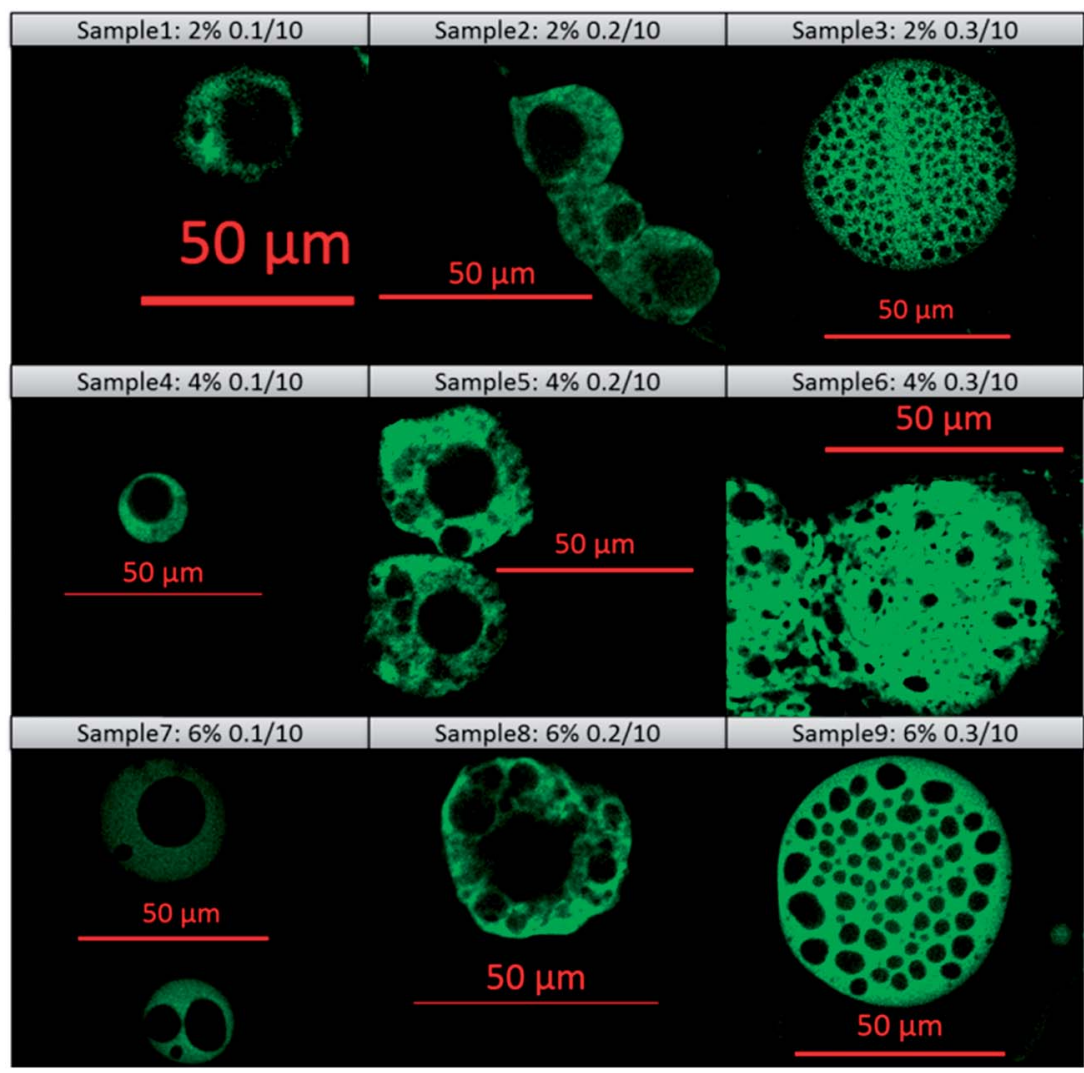

Fig. 6 The internal structure of hollow zein microcapsules produced at different concentrations of zein and flow rate combinations. Sample code: zein\% dispersing phase flow rate $\left(\mathrm{ml} \mathrm{h}^{-1}\right)$ /continuous phase flow rate $\left(\mathrm{ml} \mathrm{h}^{-1}\right)$. The scale bar for each image is $50 \mu \mathrm{m}$

\section{Morphology and internal structures of zein microcapsules}

The morphologies of the dried particles at various zein concentrations and flow rate combinations are shown in Fig. 4. One of the promising features of microfluidic devices is that the droplet size can be finely tuned by adjusting the flow rates of the dispersing and continuous phases. ${ }^{35}$ Image analysis was performed to calculate the particle sizes of the microcapsules, and the results are shown in Fig. 5. A trend was identified that the size of the microcapsule increased with an increasing flow rate of the dispersing phase, while the zein concentration had less impact. The flow rate ratio is one of the predominant factors governing the droplet size, which has been described in a previous study. ${ }^{36}$ On the other hand, the zein concentration in the dispersing phase determined the solid content in the droplet, and the droplets with greater amounts of zein are more resistant against shrinking during ethanol diffusion, which also resulted in larger microcapsule sizes.

Precise control over particle size generally correlates with enhanced control of the rate of release of an encapsulant. It was observed from the SEM images that the particle size was relatively uniform at low dispersing flow rates ( 0.1 and $0.2 \mathrm{ml} \mathrm{h}^{-1}$ ), but a larger variation of particle size was observed when the flow rate of the dispersing phase reached $0.3 \mathrm{ml} \mathrm{h}^{-1}$. A probable reason is that the parallel flow extended too far in the microfluidic channel when the flow rate of the dispersing phase increased. Therefore the detachment occurred at later time, leaving a short residence time for the zein to self-assemble. Without a completely hardened droplet surface, microcapsules may coalesce in the tubing or collector, resulting in nonuniform particle size. There are two other reasons that might also cause the polydispersity of protein capsules in this study. First, zein is a biologically-originated material, which is naturally inhomogeneous. Zein contains $\alpha$-zein, $\beta$-zein, $\gamma$-zein and $\delta$ zein, and these conformations have the tendency to form micelles or nanoaggregates in solution and therefore alter the local concentrations of each composition. ${ }^{40}$ Another reason could be related to the morphological change during the drying process. When water is removed from the core, different degrees of shrinkage could occur which may result in different shapes and sizes. For example, bowl-like and wrinkled structures have been found, depending on the hollow core distribution patterns.

Fig. 6 displays the internal structure of zein microcapsules captured using CLSM. The images show that the internal structure of the microcapsules was hollow, and the number of hollow cores varied from a single core to multiple cores by simply adjusting the flow rate of the dispersing phase. At a lower flow rate of the dispersing phase, zein microcapsules with fewer hollow cores were formed, while a higher dispersing flow rate tended to form a larger number of hollow cores. The differences in the number of hollow cores could be attributed to the droplet size, which could affect the rate of ethanol diffusion during the internal phase separation process. When the droplet 

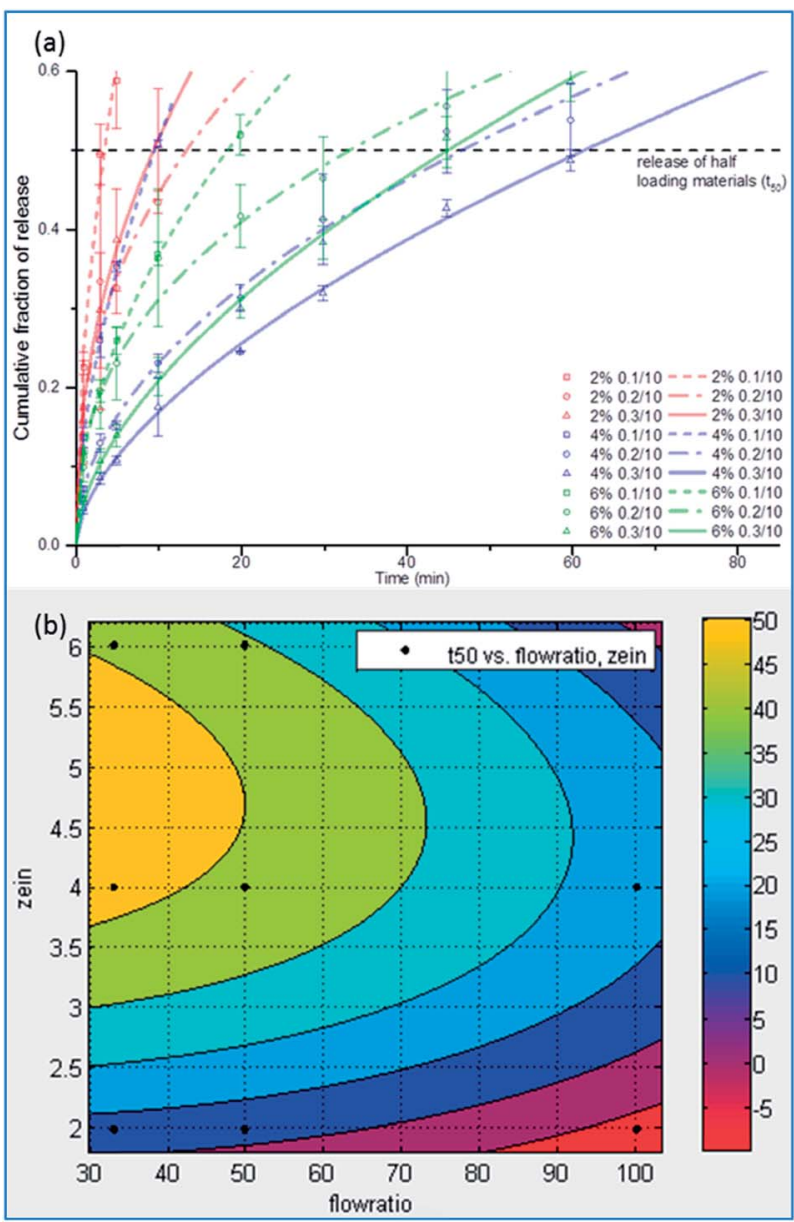

Fig. 7 Release performance of the zein microcapsules prepared at different conditions. (a) The combined release curves of all nine samples, displaying normalized fractional release versus release time (min) and fitting curves. Sample code: zein\% dispersing phase flow rate $\left(\mathrm{ml} \mathrm{h}^{-1}\right)$ /continuous phase flow rate $\left(\mathrm{ml} \mathrm{h}^{-1}\right)$. (b) The response surface plot of the release half-life time as a function of the zein concentration $(\%, w / v)$ and flow rate ratio.

size was small, ethanol could be readily extracted into the continuous phase, tributyrin, leaving a large but single hollow core, while the slow diffusion of ethanol to the continuous phase was expected in the case of large droplets. The formation of internal structure is difficult to predict due to the complexity of two competing processes: ethanol diffusion and zein selfassembly. The rate of either process could have a significant impact on the other, for example, the formation of a zein network could decrease the rate of ethanol diffusion. Therefore, the general conclusion of a positive correlation between the flow rate of the dispersing phase and the number of hollow cores may not be drawn. However, this method did provide a facile approach to tune the internal structure of the microcapsules.

\section{Release profiles}

Fig. 7(a) shows the release profiles and fitted curves of all of the samples tested in this study. The release test was carried out for 24 hours and approximately $60 \%$ of Rhodamine was recovered. This number is reasonable because Rhodamine B has a greater affinity for ethanol than water and a considerable fraction of Rhodamine B was diffused along with ethanol.

The samples produced with $2 \%$ zein released the dye fairly quickly, probably due to the formation of thinner walls than the samples with higher zein concentrations. Increasing the zein concentration to $4 \%$ was suitable to prolong the release while increasing the zein concentration to $6 \%$ did not induce further delay of the release. The cross-section images indicate that the difference in pore size between the $4 \%$ and $6 \%$ zein samples might not be significant, so it is reasonable to conclude that a further increase in the zein concentration may not further modify the release profile. On the other hand, increasing the flow rate of the dispersing phase could also prolong the release. It has been discussed above that a larger size of microcapsules was generated at higher dispersing flow rates. Usually, larger particles possess smaller surface-to-volume ratios, so the release of the dye is hindered as well. Another reason could be associated with the structure of the microcapsules. Those microcapsules with single large cores could release the core material faster than those microcapsules with multiple small pores, which could be attributed to the differences in wall thickness. For the microcapsules with single large cores, the wall was thin, which favors fast diffusion. However, for those microcapsules with a porous structure with multiple small cores, the pores embedded in the center had multiple relatively thick walls to pass through during diffusion. A similar finding and mechanism were also reported in a previous study with quantifications. ${ }^{41}$ When releasing drugs in in vitro models, the rate of release is more complicated and also depends on other factors such as degradation of microcapsules. ${ }^{42}$ Therefore, further development and optimization is necessary to engineer the microcapsule for applications such as gastro-intestinal delivery. Response surface is a robust methodology to study multivariable experiments and to find the experimental region that would generate desirable results. ${ }^{43}$ Fig. 7(b) shows a response surface that correlates the release half-lives $\left(t_{50}\right)$ with zein concentrations and flow rates $\left(R^{2}=0.852\right)$. Based on the response surface prediction, a wide range of $t_{50}$, from 3 minutes to 62 minutes, was achieved by adjusting the zein concentrations and flow rate ratios. Microcapsules that are capable of sustained release could be used as intestinal delivery carriers for enhanced bioavailability, or encapsulating antimicrobial components and fragrances for extended efficacy. ${ }^{\mathbf{4 4 5}}$ On the other hand, microcapsules with boosted release properties could be used for flavor compounds in order to rapidly release flavors during oral processing while improving flavor stability during storage. ${ }^{46}$

\section{Conclusions}

In this study, we demonstrated a facile approach to fabricating hollow protein microcapsules in a T-junction microfluidic chip. It is a single-step process that features great biocompatibility and all of the materials involved were non-hazardous. The generation of droplets in the microfluidic chip allowed the creation of an interface to direct zein self-assembly and hence the formation of microcapsules. By adjusting the zein 
concentration in the dispersing phase and flow rates during processing, microcapsules with various particle sizes, internal structures and permeabilities could be prepared, which will allow for the design of microcapsules that are versatile for various applications. This type of microcapsule can be an alternative for replacing colloidosomes. It is easier, safer and more cost-effective to produce than colloidosomes. Furthermore, this study also presented the great potential of zein as a food-grade protein, which can be incorporated for expanded applications.

\section{Conflicts of interest}

There are no conflicts to declare.

\section{Acknowledgements}

This work was partially supported by the Hatch Project No. ILLU-698-380, United State Department of Agriculture.

\section{References}

1 S. Gouin, Trends Food Sci. Technol., 2004, 15, 330-347.

2 G. Kaufman, S. Nejati, R. Sarfati, R. Boltyanskiy, M. Loewenberg, E. R. Dufresne and C. O. Osuji, Soft Matter, 2015, 11, 7478-7482.

3 K. L. Thompson, M. Williams and S. P. Armes, J. Colloid Interface Sci., 2014, 447, 217-228.

4 D. Lee and D. A. Weitz, Adv. Mater., 2008, 20, 3498-3503.

5 A. D. Dinsmore, M. F. Hsu, M. G. Nikolaides, M. Marquez, A. R. Bausch and D. A. Weitz, Science, 2002, 298, 1006-1009.

6 A. San Miguel, J. Scrimgeour, J. E. Curtis and S. H. Behrens, Soft Matter, 2010, 6, 3163.

7 D. Mark, S. Haeberle, G. Roth, F. von Stetten and R. Zengerle, Chem. Soc. Rev., 2010, 39, 1153.

8 D. M. Headen, G. Aubry, H. Lu and A. J. García, Adv. Mater., 2014, 26, 3003-3008.

9 A. G. Olenskyj, Y. Feng and Y. Lee, J. Food Eng., 2017, 211, 5059.

10 B. Kim, T. Y. Jeon, Y.-K. Oh and S.-H. Kim, Langmuir, 2015, 31, 6027-6034.

11 H. C. Shum, A. R. Abate, D. Lee, A. R. Studart, B. Wang, C. H. Chen, J. Thiele, R. K. Shah, A. Krummel and D. A. Weitz, Macromol. Rapid Commun., 2010, 31, 108-118.

12 J. Zhang, R. J. Coulston, S. T. Jones, J. Geng, O. A. Scherman and C. Abell, Science, 2012, 335, 690-694.

13 V. L. Workman, S. B. Dunnett, P. Kille and D. D. Palmer, Macromol. Rapid Commun., 2008, 29, 165-170.

14 Y. Zheng, Z. Yu, R. M. Parker, Y. Wu, C. Abell and O. A. Scherman, Nat. Commun., 2014, 5, 5772.

15 J. Zhou, D. C. Hyun, H. Liu, H. Wu and Y. Xia, Macromol. Rapid Commun., 2014, 35, 1436-1442.

16 H. Zhang, E. Tumarkin, R. M. A. Sullan, G. C. Walker and E. Kumacheva, Macromol. Rapid Commun., 2007, 28, 527538.

17 C. X. Zhao and A. P. J. Middelberg, Microfluid. Nanofluid., 2013, 14, 703-709.
18 C. X. Zhao and A. P. J. Middelberg, Angew. Chem., Int. Ed., 2009, 48, 7208-7211.

19 Y. Zhang, L. Cui, X. Che, H. Zhang, N. Shi, C. Li, Y. Chen and W. Kong, J. Controlled Release, 2015, 206, 203-219.

20 K. Pan and Q. Zhong, Annu. Rev. Food Sci. Technol., 2016, 7, 245-266.

21 K. Pan and Q. Zhong, Food Hydrocolloids, 2016, 52, 600-606. 22 Y. Feng and Y. Lee, Food Hydrocolloids, 2016, 56, 292-302.

23 Y. Wang and G. W. Padua, Langmuir, 2012, 28, 2429-2435.

24 Q. Zhong and M. Jin, Food Hydrocolloids, 2009, 23, 23802387.

25 T. Brugarolas, F. Q. Tu and D. Lee, Soft Matter, 2013, 9, 90469058.

26 H. Chen and Q. Zhong, Food Hydrocolloids, 2015, 43, 593602.

$27 \mathrm{~S} . \mathrm{Hu}, \mathrm{T}$. Wang, M. L. Fernandez and Y. Luo, Food Hydrocolloids, 2016, 61, 821-831.

28 Y. Luo, B. Zhang, M. Whent, L. L. Yu and Q. Wang, Colloids Surf., B, 2011, 85, 145-152.

29 G. Golomb, P. Fisher and E. Rahamim, J. Controlled Release, 1990, 12, 121-132.

30 A. Micsonai, F. Wien, L. Kernya, Y.-H. Lee, Y. Goto, M. Réfrégiers and J. Kardos, Proc. Natl. Acad. Sci. U. S. A., 2015, 112, E3095-E3103.

31 N. J. Greenfield, Nat. Protoc., 2006, 1, 2876-2890.

32 H. Chen, Y. Zhang and Q. Zhong, J. Food Eng., 2015, 144, 93102.

33 X. Wang, Z. Li, Y. Yang, X. Gong, Y. Liao and X. Xie, Langmuir, 2015, 31, 5456-5463.

34 R. W. Korsmeyer, R. Gurny, E. Doelker, P. Buri and N. A. Peppas, Int. J. Pharm., 1983, 15, 25-35.

35 A. S. Utada, A. Fernandez-Nieves, H. A. Stone and D. A. Weitz, Phys. Rev. Lett., 2007, 99, 94502.

36 K. Muijlwijk, C. Berton-carabin and K. Schroën, Trends Food Sci. Technol., 2016, 49, 51-63.

37 Y. Wang, F. Lopes Filho, P. Geil and G. W. Padua, Macromol. Biosci., 2005, 5, 1200-1208.

38 G. Liu, D. Wei, H. Wang, Y. Hu and Y. Jiang, Chem. Eng. J., 2016, 284, 1094-1105.

39 Z. Zhang, R. Zhang, Q. Tong, E. A. Decker and D. J. McClements, Food Res. Int., 2015, 69, 274-280.

$40 \mathrm{~S}$. Kim and J. Xu, J. Cereal Sci., 2008, 47, 1-5.

41 K. A. Mehta, M. S. Kislalioglu, W. Phuapradit, A. W. Malick and N. H. Shah, J. Controlled Release, 2000, 63, 201-211.

42 D. Klose, F. Siepmann, K. Elkharraz, S. Krenzlin and J. Siepmann, Int. J. Pharm., 2006, 314, 198-206.

43 P. Z. Hou and J. Regenstein, J. Food Sci., 2006, 69, C393C398.

44 X.-Y. Shi and T.-W. Tan, Biomaterials, 2002, 23, 4469-4473. 45 P. Sarkar, A. K. Bhunia and Y. Yao, J. Food Sci., 2016, 81, M2982-M2987.

46 H. Yoshii, A. Soottitantawat, X. D. Liu, T. Atarashi, T. Furuta, S. Aishima, M. Ohgawara and P. Linko, Innovative Food Sci. Emerging Technol., 2001, 2, 55-61. 